\title{
УДК 622.276 .63 \\ КОМПЛЕКСНЫЙ ПОДХОД К ОЦЕНКЕ ЭФФЕКТИВНОСТИ ПРОЕКТИРУЕМОЙ СОЛЯНО-КИСЛОТНОЙ ОБРАБОТКИ С ИСПОЛЬЗОВАНИЕМ РЕЗУЛЬТАТОВ ЛАБОРАТОРНЫХ ИССЛЕДОВАНИЙ И ГИДРОДИНАМИЧЕСКОГО МОДЕЛИРОВАНИЯ
}

\author{
Кривощеков Сергей Николаевич1, \\ Krivoshchekov@gmail.com
}

\author{
Козырев Никита Дмитриевич1,2, \\ Nikita.Kozyrev@pnn.lukoil.com \\ Вяткин Кирилл Андреевич ${ }^{1}$, \\ Kirill.Vyatkin@girngm.ru \\ Равелев Кирилл Алексеевич1, \\ Kirill.Ravelev@pstu.ru \\ 1 Пермский национальный исследовательский политехнический университет, \\ Россия, 614990, г. Пермь, пр. Комсомольский, 29. \\ 2 Филиал ООО «ЛУКОЙЛ-Инжиниринг» «ПермНИПИнефть» в г. Перми, \\ Россия, 614000, г. Пермь, ул. Советской Армии, 29.
}

Актуальность научной работы обусловлена тем, что в настоящее время высокая доля соляно-кислотных обработок не обеспечивают ожидаемого результата на нефтяных месторождениях Пермского края. Данная проблема непосредственно связана с действующим подходом к проектированию технологических операций по стимуляции притока нефти и недостаточной изученностью большинства факторов, существенно влияющих на эффективность обработок призабойной зоны кислотными составами. Востребованность усовершенствования технологий кислотного воздействия на призабойную зону карбонатных коллекторов и способов прогнозирования его эфффективности обуславливается низкой себестоимостью проведения данной операции и возможностью достижения высоких показателей продуктивности скважины при качественном проектировании и проведении операции.

Цель: разработка методического подхода к прогнозированию технологического эфффекта проектируемой соляно-кислотной обработки на основе комбинирования результатов лабораторных исследований и гидродинамического моделирования.

Объектами исследования являются технологии повышения нефтеотдачи пластов, литологически представленных карбонатными разностями. В пределах основных нефтегазоносных провинции России большая часть месторождений находится на завершающей стадии разработки, вследствие чего отмечается низкая продуктивность скважин. Поэтому совершенствование методов увеличения нефтеотдачи является важным и актуальным направлением исследования в нефттегазовой отрасли.

Методы: лабораторные исследования, заключающиеся в определении карбонатности изучаемых отложений, а также свойств кислотных составов, применяемых при проведении соляно-кислотных обработок на месторождениях Пермского края. Данные исследования необходимы для установления численного значения изменения скин-фактора на рассматриваемых объектах. Гидродинамическое моделирование с учетом данного скин-фрактора для определения технологической эффрективности проектируемой технологии обработки призабойной зоны продуктивного пласта.

Результаты. По итогам настоящего исследования отмечается высокое соответствие фактических изменений скинфактора на 18 объектах после кислотного воздействия, полученных при анализе баз данных гидродинамических исследований скважин на территории Пермского края, и прогнозируемых значений по описанной методике предварительной оценки технологического эфрфекта от соляно-кислотной обработки. Приведен сравнительный анализ фрактических значений дебита целевой скважины, зафиксированных в течение пяти месяцев эксплуатации, и прогнозируемых с помощью гидродинамического моделирования полностью описанного дизайна кислотного воздействия, в основе которого заложено расчетное значение изменения скин-фрактора. По результатам анализа получено, что предлагаемый комплексный подход к прогнозированию эфффективности соляно-кислотной обработки имеет небольшую погрешность в пределах 5 \%. С использованием гидродинамической модели рассчитан технологический эфффект спроектированной кислотной обработки на целевом объекте до 2030 г. и сделан вывод об успешности применения данного геолого-технического мероприятия. С внедрением предлагаемого комплексного подхода в нефтяные компании возможно увеличение результативности мероприятий по повышению нефтеотдачи пластов.

\section{Ключевые слова:}

Соляно-кислотная обработка, комплексный подход, гидродинамический симулятор,

скин-фрактор, эфрфективность мероприятия.

\section{Введение}

На всем протяжении эксплуатации скважины состояние призабойной зоны пласта (ПЗП) ухудшается вследствие кольматации каналов фильтрации различными частицами. Загрязненная зона вблизи скважины снижает гидродинамическую связь с пластом, тем самым продуктивность скважины не достигает возможного максимального значения, тогда как карбонатные породы и без этого характеризуются низкими фильтрационными свойствами. Необходимость детального 
изучения свойств и методов разработки карбонатных коллекторов обуславливается наличием в них от одной трети до половины запасов углеводородного сырья по всему миру [1].

Наиболее эффективным и экономически выгодным методом интенсификации добычи нефти при ухудшенном состоянии околоскважинной зоны пласта (ОЗП) на сегодняшний день является солянокислотная обработка (СКО) [2-6]. Кислотное воздействие не всегда достигает планируемых результатов вследствие недоученности таких ключевых факторов, как фильтрационных свойств пласта, структуры ПЗП и ее параметров до стимуляции скважины, а также их характера поведения в процессе обработки кислотным составом (КС).

Успешность проведения химического воздействия на ОЗП характеризуется отрицательным изменением скин-фактора $S$, который принято рассчитывать по формуле Hawkins (1) [7]:

$$
S=\left(\frac{k}{k_{d}}-1\right) \ln \frac{r_{d}}{r_{w}},
$$

где $k$ - коэффициент проницаемости удаленной зоны пласта; $k_{d}$ - коэффициент проницаемости приближенной зоны пласта; $r_{d}$ - радиус ПЗП; $r_{w}$ - радиус скважины.

Выражение (1) учитывает различие проницаемостей приближенной и удаленной зон пласта, за счет чего производится оценка степени загрязнения ПЗП.

Процесс кислотной обработки считается достаточно сложным и необъяснимым в деталях протекания растворения соляной кислотой минералов карбонатных пород-коллекторов. Поэтому при прогнозировании технологического эффекта от рассматриваемого метода по интенсификации добычи углеводородов возникает множество проблем [8], связанных с невозможностью предсказания характера изменения пустотного пространства продуктивного пласта вблизи скважины. В настоящее время ученые стремятся найти способ решения этих проблем, одним из них является проведение фильтрационных исследований на керне при пластовых условиях [9-11]. С помощью компьютерной томографии определяется изменение порового пространства, а именно размеры и положение прорвавшегося сквозь образец высокопроводящего канала фильтрации, называемого «червоточиной». Однако в статье [12] авторы указывают на недостоверность результатов, полученных при проведении исследований на стандартных образцах горной выработки с использованием фильтрационной установки, по отношению к реальным кислотным обработкам на нефтепромысле, что связано с высокой анизотропией коллекторских свойств карбонатных нефтегазонасыщенных пород.

Наибольшее количество научно-исследовательских работ направлено на изучение процесса формирования «червоточин», создающихся под воздействием КС в горных породах. Авторы работы [13] провели анализ существующих моделей, описывающих данный процесс в деталях. Применение изученных моделей рассматривается в публикации [14], где использованы полуэмпирические модели Гонга, Фарая, Бьюд- жиса, позволяющие спрогнозировать направление распространения КС в поровом пространстве ПЗП и оценить прирост коэффициента проницаемости в данной зоне. Полученные фильтрационные характеристики дают возможность рассчитать дополнительный приток флюида с помощью известных формул плоскорадиального движения жидкостей к гидродинамически несовершенным скважинам за счет снижения дополнительного фильтрационного сопротивления, обеспечивающегося изменением скин-фактора.

Пример предварительной оценки эффективности СКО карбонатных горных пород также приводится в работе [15], авторы которой разработали симулятор моделирования кислотного воздействия, позволяющий рассчитать изменение структуры горной породы вблизи скважины. Программа основана на развитии «червоточины» в процессе обработки КС, в качестве модели которой использована полуэмпирическая модель, предложенная М. Buijse и G. Glasbergen [16]. Рассмотренное изобретение включает в модель все фильтрационно-емкостные свойства нефтенасыщенных пропластков и моделирует их изменение при имитировании нагнетания КС. Успешность проведения СКО также оценивается через снижение фильтрационного сопротивления в ОЗП.

Таким образом, исходя из анализа публикаций, можно сделать вывод, что существующие способы прогнозирования эффективности кислотной обработки в основном строятся на определении изменения скин-фактора, который, в свою очередь, изменяется на основании увеличения коэффициента проницаемости ПЗП. Для более точной оценки эффективности проектируемых геолого-технических мероприятий применяют гидродинамическое моделирование, позволяющее произвести расчет и оценить увеличение продуктивности скважин. В Пермском крае сегодня при проектировании дизайна кислотного воздействия на нефтедобывающих скважинах в большинстве случаев включены в основу промысловые данные с ранее проведенных геолого-технических мероприятий на этом же месторождении либо на месторожденияханалогах. Тем самым объясняется несоответствие фактических и прогнозных значений дебита нефти изза неправильного подхода к созданию модели СКО, осуществляемому при интерпретации степени изменения скин-фактора с предыдущих химических обработок коллекторов.

\section{Описание комплексного подхода к оценке эффективности проектируемой соляно-кислотной обработки}

В настоящей работе для прогнозирования эффективности СКО карбонатных отложений предлагается c помощью гидродинамического моделирования определить прирост дополнительной добычи нефти и продолжительность эффекта, основываясь на теоретических расчетах снижения скин-фактора. Эксперименты, проведенные G. Daccord и R. Lenormand и заключающиеся в исследовании взаимодействия КС и горной породы, привели к эмпирическому уравнению (2), позволяющему оценить изменение скин-фактора 
после кислотного воздействия на призабойную зону пласта [17]:

$$
\Delta S=-\frac{1}{d} \ln \left(1+N_{a c} N_{P e}^{-1 / 3} \frac{b V}{\pi h m r_{w}^{d}}\right),
$$

где $d$ - фрактальная размерность образовавшейся в ПЗП структуры порового пространства; $N_{a c}$ - кислотное число, д. ед.; $N_{P e}$ - число Пекле; $b$ - константа, принимая равной $1,7 \cdot 10^{4} \mathrm{M}^{\mathrm{d}-2} ; V$ - объем закачиваемого $\mathrm{KC}, \mathrm{m}^{3} ; h$ - перфорированная толщина пласта, м; $m-$ пористость, $\%$.

При вычислении фрактальной размерности $d$ следует учесть количественные содержания известняка и доломита в карбонатной породе. Ниже приведено выражение для определения значения фрактальной размерности (3):

$$
d=\frac{1,6 x+2 y}{x+y},
$$

где $x, y$ - содержание известняка и доломита в породе, соответственно, \%.

Для определения процентного соотношения кальцита и доломита в горной породе необходимо провести лабораторные исследования с применением прибора, например, карбонатомера КМ-04М, позволяющего определять массовое содержание кальцита, доломита и нерастворимого минерального остатка в измельченном образце породы.

Кислотное число определяется соотношением, приведенным в выражении (4):

$$
N_{a c}=\frac{m C_{\mathrm{HCl}}}{C_{\text {кол }} \vartheta},
$$

где $C_{\mathrm{HCl}}-$ концентрация соляной кислоты в кислотном составе, моль/л; $C_{\text {кол }}-$ концентрация породы коллектора, моль/л; Э- стехиометрический коэффициент породы коллектора, д. ед.

Концентрация хлороводородной кислоты вычисляется согласно данным из паспорта КС (5):

$$
C_{\mathrm{HCl}}=\frac{m_{\mathrm{HCl}} \rho_{\mathrm{KC}}}{M_{\mathrm{HCl}} z},
$$

где $m_{\mathrm{HCl}}-$ масса соляной кислоты в 100 г КС, г; $\rho_{\mathrm{KC}}-$ плотность КС, г/л; $M_{\mathrm{HCl}}-$ молярная масса $\mathrm{HCl}$, г/моль; $z$ - константа, принимаемая равной 100 г.

Значение концентрации коллектора зависит от процентных соотношений известняка и доломита в интересующей породе и определяется следующим равенством (6):

$$
C_{\text {кол }}=\frac{C_{\mathrm{CaCO}_{3}} x+C_{\mathrm{CaMg}_{\left(\mathrm{CO}_{3}\right)_{2}} y},}{x+y},
$$

где $C_{\mathrm{CaCO}_{3}}$ - концентрация чистого известняка, моль/л; $C_{\mathrm{CaMg}\left(\mathrm{CO}_{3}\right)_{2}}-$ концентрация чистого доломита, моль/л.

Для определения концентраций из справочных данных используются значения молекулярных масс пород, вступающих в реакцию, т. е. чистого известняка $M_{\mathrm{CaCO}_{3}}$, доломита $M_{\mathrm{CaMg}\left(\mathrm{CO}_{3}\right)_{2}}$. Концентрация соответствующего вещества рассчитывается по выражению (7):

$$
C=\frac{\rho}{M},
$$

где $\rho$ - плотность вещества, г/л; $M$ - молекулярная масса вещества, г/моль.

По справочным данным плотности кальцита и доломита принимаются равными 2710 и 2880 г/л, соответственно, а их молекулярные массы - 100,086 и 184,399 г/моль.

Исходя из уравнений реакций, стехиометрический коэффициент для известняка принимается равным $\vartheta_{\mathrm{CaCO}_{3}}=2$, а для доломита $\vartheta_{\mathrm{CaMg}\left(\mathrm{CO}_{3}\right)_{2}}=4$, следовательно, стехиометрический коэффициент коллектора определяется подобно фрактальной величине (8):

$$
\vartheta=\frac{\vartheta_{\mathrm{CaCO}_{3}} x+\vartheta_{{\mathrm{CaMg}\left(\mathrm{CO}_{3}\right)_{2}} y} .}{x+y}
$$

С целью определения числа Пекле необходимо воспользоваться выражением (9):

$$
N_{P e}=\frac{q}{D h},
$$

где $q$ - темп закачки кислотного состава, $\mathrm{m}^{3} / \mathrm{c} ; D$ - коэффициент диффузии, $\mathrm{m}^{2} / \mathrm{c}$.

В настоящей работе предлагается оценивать коэффициент диффузии кислоты с использованием формулы Стокса-Эйнштейна $[18,19]$, которая имеет широкое применение для определения значения $D$ в жидкостях (10):

$$
D=\frac{k T}{6 \pi \mu r}
$$

где $k=1,38 \cdot 10^{-23}$ - постоянная Больцмана, Дж/К; $T$ абсолютная температура, К; $\mu$ - коэффициент динамической вязкости КС, Па·с; $r$ - радиус диффундирующей частицы, м.

Один из вариантов определения динамической вязкости КС - это метод, используемый в соответствии с ГОСТ 33768-2015 с помощью вискозиметра типа Пинкевича (ВПЖ-4), представленный на рис. 1. Радиус диффундирующей частицы можно установить при помощи бинокулярного микроскопа.

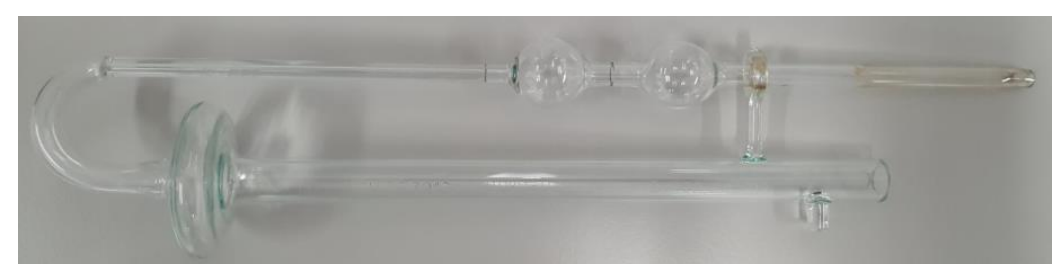

Puc. 1. Вискозиметр типа Пинкевича (ВПЖ-4)

Fig. 1. VPZh-4 viscometer 
Для прогнозирования определения эффективности соляно-кислотного воздействия рассчитанное значение изменения скин-фактора, полученное при решении выражения (2) и при проведении рассмотренного ряда лабораторных исследований, заносится в гидродинамическую модель эксплуатационного объекта, после чего производится расчет основных технологических параметров работы целевой нефтедобывающей скважины. В результате проведенных гидродинамических расчетов при помощи специализированных симуляторов выгружаются графики изменения годовой добычи нефти и воды по целевой скважине по базовому варианту и после проведения проектируемого мероприятия. На основании изменения годовой добычи нефти и воды делается заключение о прогнозной эффективности применения кислотного воздействия на целевой нефтедобывающей скважине. Статистический анализ опыта применения
соляно-кислотных обработок

на месторождениях Пермского края

Для интенсификации добычи нефти из карбонатных пластов в Пермском крае применяются СКО. С целью определения возможности практического применения выражения (2) для прогнозирования эффективности от проектируемого кислотного воздействия проведен статистический анализ. В работе рассмотрен опыт 18 технологических операций по кислотному воздействию на месторождениях Пермского края. Для каждой из рассматриваемых операций определено ожидаемое изменение скин-фактора, рассчитанное по представленной методике прогнозирования изменения скин-фактора после СКО. В табл. 1 приведено сравнение фактического изменения скин-фактора по рассматриваемым скважинам и прогнозного значения, полученного по представленной методике.

Таблица 1. Сравнение фактического изменения скин-фактора с прогнозными значениями

Table 1. Comparison of skin factor actual change with predicted values

\begin{tabular}{|c|c|c|c|c|c|}
\hline $\begin{array}{c}\text { № технологической } \\
\text { операции } \\
\text { Job no. }\end{array}$ & $\begin{array}{c}\text { Месторождение } \\
\text { Deposit }\end{array}$ & $\begin{array}{c}\text { Пласт } \\
\text { Formation }\end{array}$ & $\begin{array}{c}\text { Фактическое измене- } \\
\text { ние скин-фактора } \\
\text { Skin factor actual } \\
\text { change }\end{array}$ & $\begin{array}{c}\text { Прогнозное измене- } \\
\text { ние скин-фактора } \\
\text { Skin factor predicted } \\
\text { change }\end{array}$ & $\begin{array}{c}\text { Отклонение, \% } \\
\text { Deviation, \% }\end{array}$ \\
\hline 1 & $\begin{array}{l}\text { Озерное } \\
\text { Ozernoe }\end{array}$ & $\begin{array}{c}\text { Бш-Срп } \\
\text { Bashkirian- } \\
\text { Serpukhovian } \\
\end{array}$ & $-3,40$ & $-3,27$ & 3,82 \\
\hline 2 & $\begin{array}{l}\text { Уньвинское } \\
\text { Unvinskoe }\end{array}$ & $\begin{array}{c}\text { Бш-Срп } \\
\text { Bashkirian- } \\
\text { Serpukhovian } \\
\end{array}$ & $-2,80$ & $-2,90$ & 3,57 \\
\hline 3 & $\begin{array}{l}\text { Змеевское } \\
\text { Zmeevskoe }\end{array}$ & $\begin{array}{c}\mathrm{T} \\
\text { Tournaisian } \\
\end{array}$ & $-3,20$ & $-2,94$ & 8,13 \\
\hline 4 & $\begin{array}{l}\text { Юрчукское } \\
\text { Yurchukskoe }\end{array}$ & $\begin{array}{c}\Phi_{M} \\
\text { Famennian }\end{array}$ & $-2,50$ & $-2,74$ & 9,60 \\
\hline 5 & $\begin{array}{l}\text { Юрчукское } \\
\text { Yurchukskoe }\end{array}$ & $\begin{array}{c}\text { Бш } \\
\text { Bashkirian }\end{array}$ & $-2,70$ & $-2,53$ & 6,30 \\
\hline 6 & $\begin{array}{l}\text { Логовское } \\
\text { Logovskoe }\end{array}$ & $\begin{array}{c}\text { T- } \Phi_{M} \\
\text { Tournaisian- } \\
\text { Famennian } \\
\end{array}$ & $-2,60$ & $-2,84$ & 9,23 \\
\hline 7 & $\begin{array}{l}\text { Павловское } \\
\text { Pavlovskoe }\end{array}$ & $\begin{array}{c}\mathrm{T} \\
\text { Tournaisian } \\
\end{array}$ & $-2,40$ & $-2,57$ & 7,08 \\
\hline 8 & $\begin{array}{l}\text { Шагиртско- } \\
\text { Гожанское } \\
\text { Shagirtsko- } \\
\text { Gozhanskoe }\end{array}$ & $\begin{array}{c}\mathrm{B}_{3} \mathrm{~B}_{4} \\
\text { Vereiskian } \\
(\mathrm{V} 3 \mathrm{~V} 4)\end{array}$ & $-2,90$ & $-2,71$ & 6,55 \\
\hline 9 & $\begin{array}{l}\text { Дороховское } \\
\text { Dorokhovskoe }\end{array}$ & $\begin{array}{c}\mathrm{T} \\
\text { Tournaisian }\end{array}$ & $-2,80$ & $-2,83$ & 1,07 \\
\hline 10 & $\begin{array}{l}\text { Павловское } \\
\text { Pavlovskoe }\end{array}$ & $\begin{array}{c}\mathrm{T} \\
\text { Tournaisian }\end{array}$ & $-3,00$ & $-2,96$ & 1,33 \\
\hline 11 & $\begin{array}{l}\text { Сибирское } \\
\text { Sibirskoe }\end{array}$ & $\begin{array}{c}\text { Бш-Срп } \\
\text { Bashkirian- } \\
\text { Serpukhovian } \\
\end{array}$ & $-2,90$ & $-2,70$ & 6,90 \\
\hline 12 & $\begin{array}{l}\text { Чураковское } \\
\text { Churakovskoe }\end{array}$ & $\begin{array}{c}\mathrm{T} \\
\text { Tournaisian }\end{array}$ & $-2,80$ & $-2,58$ & 7,86 \\
\hline 13 & $\begin{array}{l}\text { Рассветное } \\
\text { Rassvetnoe }\end{array}$ & $\begin{array}{c}\text { Бш } \\
\text { Bashkirian }\end{array}$ & $-3,10$ & $-3,26$ & 5,16 \\
\hline 14 & $\begin{array}{l}\text { Рассветное } \\
\text { Rassvetnoe }\end{array}$ & $\begin{array}{c}\text { БШ } \\
\text { Bashkirian }\end{array}$ & $-2,50$ & $-2,79$ & 11,60 \\
\hline 15 & $\begin{array}{c}\text { Курбатовское } \\
\text { Kurbatovskoe } \\
\end{array}$ & $\begin{array}{c}\mathrm{T} \\
\text { Tournaisian } \\
\end{array}$ & $-2,60$ & $-2,77$ & 6,54 \\
\hline 16 & $\begin{array}{l}\text { Юрчукское } \\
\text { Yurchukskoe }\end{array}$ & $\begin{array}{c}\text { Бш-Срп } \\
\text { Bashkirian- } \\
\text { Serpukhovian }\end{array}$ & $-2,80$ & $-2,68$ & 4,29 \\
\hline 17 & $\begin{array}{l}\text { Юрчукское } \\
\text { Yurchukskoe }\end{array}$ & $\begin{array}{c}\text { Бш-Срп } \\
\text { Bashkirian- } \\
\text { Serpukhovian }\end{array}$ & $-2,90$ & $-2,64$ & 8,97 \\
\hline 18 & $\begin{array}{c}\text { Чикулаевское } \\
\text { Chikulayevskoe }\end{array}$ & $\begin{array}{c}\mathrm{T} \\
\text { Tournaisian }\end{array}$ & $-2,70$ & $-2,85$ & 5,56 \\
\hline
\end{tabular}


Как видно, относительные погрешности, представленные в табл. 1, изменяются в диапазоне от 1,07 до $11,60 \%$, что является вполне допустимым отклонением. Для более точного анализа и установления значимости связи между двумя совокупностями значений, т. е. фактическими и рассчитанными изменениями скин-фактора, необходимо воспользоваться основными статистическими показателями. В ходе проведения расчетов с помощью программного продукта STATISTICA получено, что критерий Стьюдента и достигаемый уровень значимости составляют 0,079 и 0,937, соответственно, а стандартные отклонения также не выходят за рамки допустимых значений, т. е. разброс значений выборки относительно среднего незначителен. Проведенный анализ позволяет сказать о большой значимости связи между фактическими и проектными данными, что свидетельствует об эффективном подходе к прогнозированию результатов СКО с использованием предложенной методики.

Сравнительный анализ реальных и проектных результатов подвел авторов настоящей работы к уверенности в идее об использовании в основе прогнозирования успешности кислотной обработки формулы Даккорда и Ленорманда. Пример практического применения предложенного комплексного подхода на действующей скважине, заключающегося в определении эффективности проведения СКО при помощи гидродинамического моделирования, приведен в настоящей работе. А также оценены отклонения от фактических данных, приведенных за пять месяцев эксплуатации после кислотного воздействия на нефтенасыщенный пласт.

\section{Дизайн соляно-кислотного воздействия \\ на целевом объекте и прогнозная оценка эффективности мероприятия с применением гидродинамического моделирования}

Целевым объектом в марте 2020 г. для проведения СКО определена нефтедобывающая скважина Кокуйского нефтяного месторождения Пермского края, вскрывающая турнейский пласт. С целью установления совершенства предложенного подхода к прогнозированию эффективности кислотного воздействия авторами данного исследования приведено сравнение фактических и проектных показателей добычи за последующие пять месяцев эксплуатации рассматриваемой скважины. А также с помощью программного продукта Tempest (Roxar), позволяющего сформировать дизайн геолого-технического мероприятия, проанализирован технологический эффект от запланированной кислотной обработки ПЗП на ближайшие десять лет.

В качестве реагента подобран кислотный состав «ФЛАКСОКОР-210», отлично зарекомендовавший себя по промысловому опыту применения методов повышения нефтеотдачи пластов и показавший хорошие результаты в лабораторных исследованиях, представленных в работе [20]. По результатам гидродинамических исследований целевой нефтедобывающей скважины, проведенных за 4 месяца до кислот- ного воздействия, установлено значение скинфактора, равное -1,4. Авторами данной работы был проведен расчет снижения фильтрационного сопротивления в околоскважинной зоне пласта по представленной выше методике, позволяющий в дальнейшем на гидродинамической модели рассчитать увеличение дебита целевой скважины после обработки кислотным составом.

Перед кислотным воздействием на продуктивный пласт для устранения нежелательных последствий целесообразным является проведение лабораторных исследований по совместимости КС с флюидами, поступающими с целевого объекта. Изучение их взаимодействия играет важную роль при дизайне СКО, так как в случае плохой совместимости возможно образование высоковязкой эмульсии, сгустков, хлопьев, осадков, приводящих к кольматации пустотного пространства. С этой целью в лабораторных условиях выполнено тестирование с 15 различными вариантами соотношений нефти, воды и кислотного состава. Вначале достигалось возникновение стабильной водонефтяной эмульсии из отобранных проб нефти и воды с рассматриваемой целевой скважины, после чего в полученную эмульсию добавлялся тестируемый КС. В результате состояние полученной смеси оценивалось визуально на наличие осадка и расслоения фаз при выдержке в течение нескольких минут, а также полученная смесь фильтровалась через сито с ячейкой 100 меш с фиксацией присутствия/отсутствия не фильтруемого остатка. В табл. 2 приведен пример некоторых результатов проведенных лабораторных исследований по изучению совместимости тестируемого КС с флюидами, отобранными с целевой скважины.

В результате проведения лабораторных исследований по изучению совместимости пластовых флюидов с КС можно сделать вывод о хорошей совместимости пластовых жидкостей и кислотной композиции, поскольку во всех случаях отмечается отсутствие каких-либо признаков образования остатков при фильтрации полученных смесей. Следовательно, состав «ФЛАКСОКОР-210 марки Р» можно считать применимым к данным условиям по характеру взаимодействия с флюидами. Изучая паспорт данного КС, определена массовая доля хлористого водорода, составляющая $12,7 \%$, которая находится в допустимых пределах по ТУ 2122-075-53501222-2009.

Для определения прогнозного изменения скинфактора по выражению (2) проведен ряд лабораторных исследований для получения исходных данных для расчета. Таким образом, получены такие данные, как массовые доли кальцита и доломита, содержащиеся в горной породе (89,7 и 1,2 \%, соответственно), что характерно для турнейского яруса на территории Пермского края [21]. Значение вязкости кислотного состава «ФЛАКСОКОР-210», определенное как среднее из нескольких проведенных экспериментов, составило 1,37 мПа·с. Радиус диффундирующей частицы КС, установленный на микроскопе «Микмед-5», составил 1 мкм.

Информация о пластовом давлении дает понимание о требуемом темпе закачки кислоты в пласт. 
Процесс СКО происходит зачастую с использованием насосного агрегата АзИНмаш-30, технические характеристики которого приведены в табл. 3 [22, 23].

Таблица 2. Результать исследования совместимости пластовых флюидов, отобранных с иелевой скважины, с тестируемым кислотным составом

Table 2. Results of study of compatibility of formation fluids collected from the target well, with the acid composition under test

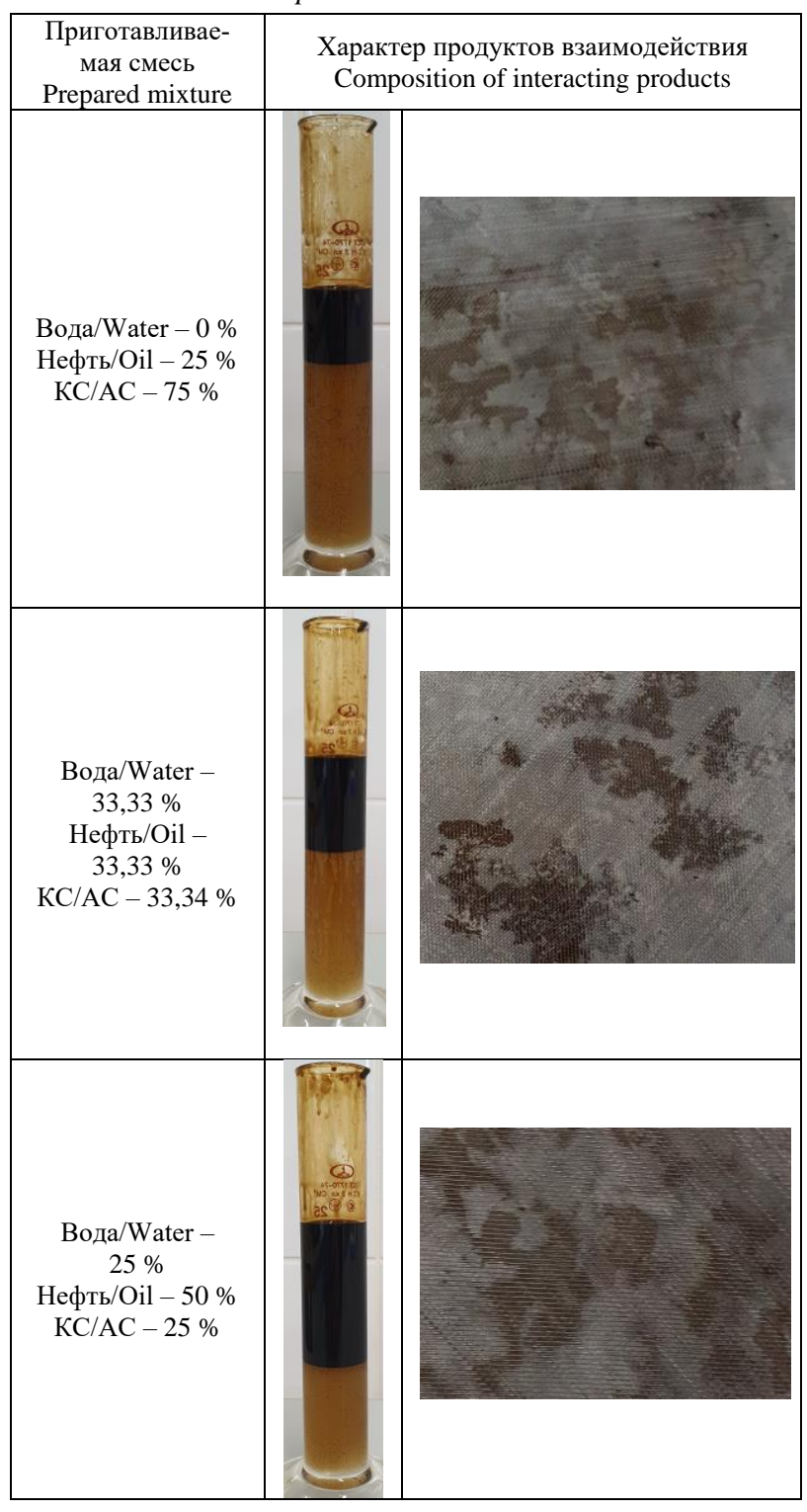

Согласно табл. 3 темп закачки кислотного состава определен равным 9,36 л/с. Температура на забое скважины, установленная с данных систем телеметрии, равна $28{ }^{\circ} \mathrm{C}$. Диаметр скважины составляет 146 мм.

Пласт, эксплуатирующийся целевой нефтедобывающей скважиной, по проведенным гидродинамическим исследованиям характеризуется сильной неоднородностью и малой проницаемостью. Вследствие этого рекомендуется применить большеобъемную соляно-кислотную обработку (БСКО), что позволит до- стичь наилучшего эффекта. В работе [24] сообщается о том, что объем кислотного состава на 1 м интервала обработки варьируется в диапазоне 3-5 м ${ }^{3}$ при использовании технологии БСКО карбонатных отложений. Следовательно, исходя из значительной вскрытой толщины пласта, было принято решение - закачивать кислоту объемом 45 м $^{3}$ для максимально возможного увеличения проницаемости ПЗП.

Таблица 3. Производительность и давление, развиваемые агрегатом типа АзИНмам-30

Table 3. Pumping rate and pressure developed by AzINmash-30 unit

\begin{tabular}{|c|c|c|c|c|}
\hline \multirow{3}{*}{ 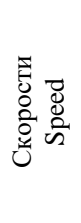 } & \multicolumn{4}{|c|}{$\begin{array}{l}\text { Диаметр плунжера, мм } \\
\text { Plunger diameter, mm }\end{array}$} \\
\hline & \multicolumn{2}{|c|}{100} & \multicolumn{2}{|l|}{120} \\
\hline & $\begin{array}{c}\text { Производи- } \\
\text { тельность, л/c } \\
\text { Pumping rate, } 1 / \mathrm{s}\end{array}$ & $\begin{array}{c}\text { Давление, } \\
\text { МПа } \\
\text { Pressure, } \\
\text { МРa }\end{array}$ & $\begin{array}{c}\text { Производи- } \\
\text { тельность, л/c } \\
\text { Pumping rate, 1/s }\end{array}$ & $\begin{array}{c}\text { Давление, } \\
\text { МПа } \\
\text { Pressure, } \\
\text { МРа }\end{array}$ \\
\hline II & 2,24 & 50,0 & 3,23 & 34,4 \\
\hline III & 4,28 & 25,9 & 6,16 & 18,0 \\
\hline IV & 6,50 & 17,1 & 9,36 & 11,8 \\
\hline $\mathrm{V}$ & 9,78 & 11,3 & 14,08 & 1,9 \\
\hline
\end{tabular}

Все полученные значения параметров, влияющие на эффективность проведения СКО, анализируются, и с помощью вычислений в конечном итоге определяется $\Delta S$ по выражению (2). В результате прогнозное уменьшение скин-фактора составляет 2,4, что несет существенное влияние на изменение количества флюида, добываемого из данной скважины. Проведенный расчет по установлению прогнозного значения скин-фактора, равного $S=-1,4-24=-3,8$, после кислотного воздействия на рассматриваемом целевом объекте позволяет провести сравнительный анализ между фактическими значениями дебита нефти и жидкости, полученными в течение пяти месяцев со скважины, и прогнозируемыми с помощью гидродинамического симулятора, представленными в табл. 4. Гидродинамическая модель, соответствующая окончанию 2030 г., представлена на рис. 2.

Согласно табл. 4, наблюдается практически полное соответствие прогнозируемых дебитов нефти и жидкости его фактическим значениям, так как в среднем отклонения составляют 5,03 и 5,29, соответственно, а среднеквадратические отклонения равны 0,38 и 0,31 . Следовательно, можно отметить высокую точность прогнозирования дебитов по разработанной методике, которая позволит оценивать эффективность кислотного воздействия на продуктивные отложения, литологически представленные карбонатными разностями.

При дальнейших расчетах на гидродинамической модели можно проанализировать дополнительную добычу углеводородов на несколько лет вперед, т. е. сделать заключение об успешности метода повышения нефтеотдачи пласта. Ниже проведен анализ эффективности проектируемого мероприятия на целевом объекте в сравнении с базовым вариантом с помощью гидродинамического моделирования, результаты которого представлены в табл. 5. Расчет проводился до 2030 г. 


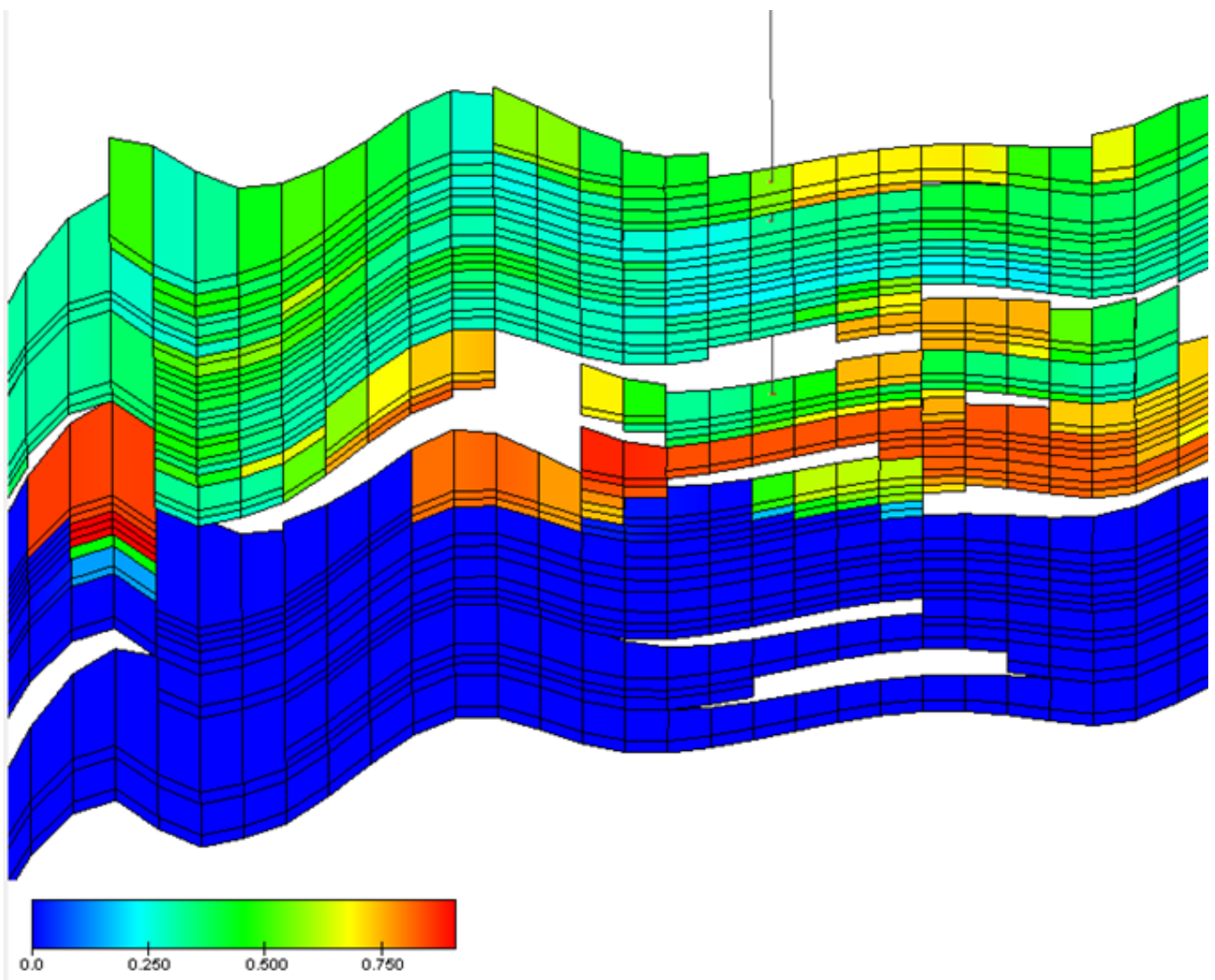

Pис. 2. Гидродинамическая модель

Fig. 2. Hydrodynamic model

Таблица 4. Сравнение фактического и прогнозируемого дебита рассматриваемой скважины

Table 4. Comparison of actual and predicted flow rates of the well under study

\begin{tabular}{|c|c|c|c|c|c|c|}
\hline \multirow{2}{*}{$\begin{array}{l}\text { Дата } \\
\text { Date }\end{array}$} & \multicolumn{2}{|c|}{$\begin{array}{l}\text { Дебит нефти, } \mathrm{m}^{3} / \text { сут } \\
\text { Oil flow rate, } \mathrm{m}^{3} / \text { day }\end{array}$} & \multirow{2}{*}{$\begin{array}{c}\text { Отклонения по дебиту } \\
\text { нефти, \% } \\
\text { Deviations in oil flow } \\
\text { rate, \% }\end{array}$} & \multicolumn{2}{|c|}{$\begin{array}{l}\text { Дебит жидкости, } \mathrm{m}^{3} / \text { сут } \\
\text { Liquid flow rate, } \mathrm{m}^{3} / \text { day }\end{array}$} & \multirow{2}{*}{$\begin{array}{c}\text { Отклонения по дебиту } \\
\text { жидкости, \% } \\
\text { Deviations in liquid flow } \\
\text { rate, \% }\end{array}$} \\
\hline & $\begin{array}{c}\text { Фактический } \\
\text { Actual }\end{array}$ & $\begin{array}{l}\text { Проектный } \\
\text { Design }\end{array}$ & & $\begin{array}{c}\text { Фактический } \\
\text { Actual }\end{array}$ & $\begin{array}{l}\text { Проектный } \\
\text { Design }\end{array}$ & \\
\hline 03.2020 & 7,4028 & 7,4028 & - & 19,2090 & 19,2090 & - \\
\hline 04.2020 & 15,9274 & 15,1492 & 4,89 & 21,1629 & 20,0937 & 5,05 \\
\hline 05.2020 & 15,6722 & 14,8356 & 5,34 & 21,1096 & 20,0741 & 4,91 \\
\hline 06.2020 & 15,4255 & 14,7529 & 4,36 & 21,0568 & 19,9116 & 5,44 \\
\hline 07.2020 & 15,1868 & 14,3710 & 5,37 & 21,0044 & 19,9007 & 5,25 \\
\hline 08.2020 & 14,9561 & 14,1783 & 5,20 & 20,9526 & 19,7418 & 5,78 \\
\hline
\end{tabular}

Таблица 5. Результаты моделирования базового и прогнозируемого вариантов

Table 5. Results of simulation of the basic and design variants

\begin{tabular}{|c|c|c|c|c|c|c|c|c|}
\hline \multirow{2}{*}{$\begin{array}{l}\text { Дата } \\
\text { Date }\end{array}$} & \multicolumn{2}{|c|}{\begin{tabular}{|l} 
Дебит нефти, $\mathrm{m}^{3} / \mathrm{cyт}$ \\
Oil flow rate, $\mathrm{m}^{3} /$ day
\end{tabular}} & \multicolumn{2}{|c|}{$\begin{array}{l}\text { Накопленная добыча нефти, тыс. } \text { м }^{3} \\
\text { Cumulative oil production, thous. } \mathrm{m}^{3}\end{array}$} & \multicolumn{2}{|c|}{$\begin{array}{l}\text { Дебит жидкости, } \mathrm{m}^{3} / \text { сут } \\
\text { Liquid flow rate, } \mathrm{m}^{3} / \text { day }\end{array}$} & \multicolumn{2}{|c|}{$\begin{array}{l}\text { Накопленная добыча жидкости, тыс. м } \\
\text { Cumulative liquid production, thous. } \mathrm{m}^{3}\end{array}$} \\
\hline & $\begin{array}{c}\text { Базовый } \\
\text { Basic }\end{array}$ & $\begin{array}{c}\text { Проект- } \\
\text { ный } \\
\text { Design }\end{array}$ & $\begin{array}{l}\text { Базовый } \\
\text { Basic }\end{array}$ & $\begin{array}{l}\text { Проектный } \\
\text { Design }\end{array}$ & $\begin{array}{l}\text { Базовый } \\
\text { Basic }\end{array}$ & $\begin{array}{l}\text { Проектный } \\
\text { Design }\end{array}$ & $\begin{array}{l}\text { Базовый } \\
\text { Basic }\end{array}$ & $\begin{array}{l}\text { Проектный } \\
\text { Design }\end{array}$ \\
\hline 01.2020 & 7,5505 & 7,5505 & 33,6657 & 33,6657 & 19,2090 & 19,2090 & 59,9883 & 59,9883 \\
\hline 01.2021 & 6,7576 & 13,8806 & 36,2659 & 36,5846 & 19,2090 & 20,6834 & 67,0188 & 67,1032 \\
\hline 01.2022 & 6,1463 & $\begin{array}{l}12,1480 \\
\end{array}$ & 38,6065 & 41,2754 & 19,2090 & 20,2897 & 74,0301 & 74,5926 \\
\hline 01.2023 & 5,6373 & 10,5697 & 40,7473 & 45,3639 & 19,2090 & 19,6713 & 81,0414 & 81,8445 \\
\hline 01.2024 & 5,1861 & 9,7903 & 42,7141 & 49,0617 & 19,2090 & 19,5334 & 88,0526 & 88,9977 \\
\hline 01.2025 & 4,7905 & 9,0952 & 44,5315 & 52,5027 & 19,2090 & 19,3793 & 95,0831 & 96,1147 \\
\hline 01.2026 & 4,4549 & 8,4170 & 46,2116 & 55,6935 & 19,2090 & 19,1928 & 102,0940 & 103,1550 \\
\hline 01.2027 & 4,1683 & 7,6169 & 47,7799 & 58,6000 & 19,2090 & 18,9866 & 109,1060 & 110,1140 \\
\hline 01.2028 & 3,9105 & 7,1231 & 49,2494 & 61,2759 & 19,2090 & 18,9334 & 116,1170 & 117,0320 \\
\hline 01.2029 & 3,6740 & 6,7406 & 50,6334 & 63,8055 & 19,2090 & 18,8832 & 123,1470 & 123,9520 \\
\hline 01.2030 & 3,4460 & 6,4022 & 51,9293 & 66,1973 & 19,2090 & 18,8363 & 130,1590 & 130,8350 \\
\hline
\end{tabular}


Анализируя данные, приведенные в табл. 4, 5, можно сделать вывод, что после проведения кислотного воздействия на целевой нефтедобывающей скважине ожидается значительный прирост добычи нефти - более чем в 2 раза. На основании реализованного прогноза после проведения СКО на целевой нефтедобывающей скважине накопленная добыча нефти к началу 2030 г. увеличится на 14,27 тыс. м³, а накопленная добыча жидкости - на 0,68 тыс. м³. Исходя из представленных величин, можно сказать об эффективности проектируемого мероприятия на выбранном целевом объекте.

\section{Заключение}

Предложенный в настоящей работе комплексный подход к определению эффективности моделируемой СКО позволяет с небольшой погрешностью прогнозировать технологическую эффективность проектируемого мероприятия. Суть методики заключается в

\section{СПИСОК ЛИТЕРАТУРЫ}

1. Исследование воздействия кислотогенерирующего состава на керны продуктивных отложений Куюмбинского лицензионного участка / Г.П. Хижняк, А.М. Амиров, Е.А. Гладких, В.А. Колесов, А.Г. Захарян, А.Е. Чикин, Р. Харрис // Нефтяное хозяйство. - 2015. - № 3. - С. 31-35.

2. Stimulation of calcite-rich shales using nanoparticlemicroencapsulated acids / R. Singh, S. Tong, K. Panthi, K. Mohanty // SPE Journal. - 2019. - V. 24 - P. 2671-2680.

3. Influence of transport conditions on optimal injection rate for acid jetting in carbonate reservoirs / D. Ridner, T. Frick, D. Zhu, A.D. Hill, R. Angeles, N. Vishnumolakala, C.E. Shuchart // SPE Production and Operations. - 2019. - V. 35. - P. 137-146.

4. A novel cationic polymer system that improves acid diversion in heterogeneous carbonate reservoirs / A. Sarmah, A. Farid Ibrahim, H.A. Nasr-El-Din, J. Jackson // SPE Oil and Gas India Conference and Exhibition. - Mumbai, 2019. - P. 2281-2295.

5. Acid stimulation improvement with the use of new particulate base diverter to improve zonal coverage in HPHT carbonate reservoirs / F. Moid, R. Rodoplu, A.M. Nutaifi, R. Kayumov // International Petroleum Technology Conference. - Dhahran, 2020. - URL: https://onepetro.org/IPTCONF/proceedings-abstract/20IPTC/320IPTC/D033S088R001/154728 (дата обращения 15.04.2021)

6. Effects of pre-existing fractures on carbonate matrix stimulation studied by large-scale radial acidizing experiments / G. Aidagulov, D. Gwaba, R. Kayumov, A. Sultan, M. Aly, X. Qiu, H. Almajed, M. Abbad // SPE Middle East Oil and Gas Show and Conference. - Manama, 2019.

7. Hawkins M.F. A note on the skin effect // Journal of Petroleum Technology. - 1956. - V. 8. - P. 65-66.

8. Ali M.T., Ezzat A.A., Nasr-El-Din H.A. A model to simulate matrix-acid stimulation for wells in dolomite reservoirs with vugs and natural fractures // SPE Journal. - 2019. - V. 25. - P. 609-631.

9. Acid stimulation-best practices for design, selection and testing of acid recipes in low permeability carbonate reservoirs / B. HallThompson, A.R. Ernesto, N. Abdulrahman, A. Alsuhaimi // International Petroleum Technology Conference. - Dhahran, 2020.

10. Integration of latest laboratory, software and retarded acid technologies to increase efficiency of acid treatments in carbonates: case studies from Central Asia / D. Abdrazakov, M. Ziauddin, D. Vernigora, A. Beletskaya, I. Yakimchuk, O. Olennikova, D. Usoltsev, M. Nikolaev, M. Panga, A. Burlibayev // International Petroleum Technology Conference. - Beijing, 2019.

11. Мартюшев Д.А. Лабораторные исследования кислотных составов для обработки коллекторов, характеризующихся различной карбонатностью и структурой пустотного пространства горных пород // Известия Томского политехнического университета. Инжиниринг георесурсов. - 2018. - Т. 329. № 4. - С. 6-12.

12. Особенности изучения фильтрационных характеристик ориентированного керна сложнопостроенных карбонатных кол- определении технологической эффективности с применением гидродинамического моделирования, в основу которого заложено расчетное значение изменения скин-фактора с использованием исходных данных, полученных в результате лабораторных исследований. Применение предлагаемого подхода позволит повысить точность технико-экономической оценки, проводимой при принятии решения о проведении геолого-технологического мероприятия. А также в методику включены параметры, с помощью которых возможно давать рекомендации по выбору кислотного состава и технологии его нагнетании в пласт для наибольшего эффекта от кислотного воздействия на карбонатные коллектора.

Исследования выполнены при финансовой поддержке Министерства науки и выстего образования Российской Федераичии (проект № FSNM-2020-0027).

лекторов / И.П. Гурбатова, В.В. Плотников, Н.А. Попов, И.В. Сысоев // Вестник Пермского национального исследовательского политехнического университета. Геология. Нефтегазовое и горное дело. - 2013. - № 9. - С. 19-28.

13. Каневская Р.Д., Новиков А.В. Методы моделирования червоточин при соляно-кислотном воздействии на карбонатные пласты // Нефтепромысловое дело. - 2018. - № 3. - С. 19-28.

14. Complex approach to the design of acid treatment of carbonate reservoirs / Y. Trushin, A. Aleshchenko, K. Danilin, A. Folomeev, A. Haydar, A. Gorin, A. Sharifullin // SPE Russian Petroleum Technology Conference. - Moscow, 2019.

15. Khuzin R., Shevko N., Melnikov S. Improving well stimulation technology based on acid stimulation modeling, lab and field data integration // SPE Russian Petroleum Technology Conference. Moscow, 2019.

16. Buijse M., Glasbergen G. A semi-empirical model to calculate wormhole growth in carbonate acidizing // SPE Annual Technical Conference and Exhibition. - Dallas, 2005. - P. 3539-3552.

17. Daccord G., Touboul E., Lenormand R. Carbonate acidizing: toward a quantitative model of the wormholing phenomenon // SPE production engineering. - 1989. - V. 4. - P. 63-68.

18. Воробьев А.X. Диффузионные задачи в химической кинетике. - М.: Изд-во Московского университета, 2003. - 98 с.

19. Chordia M., Trivedi J.J. Diffusion in naturally fractured reservoirs - a review // SPE Asia Pacific Oil and Gas Conference and Exhibition. - Brisbane, 2010. - P. 1906-1937.

20. Равелев К.А. Сравнительный анализ эффективности применения кислотных составов для проведения соляно-кислотной обработки призабойной зоны карбонатного пласта // Проблемы разработки месторождений углеводородных и рудных полезных ископаемых. - Пермь: Пермский национальный исследовательский политехнический университет, 2019. - Т. 1. C. $194-196$.

21. Потехин Д.В. Оптимизация технологии многовариантного трехмерного геологического моделирования залежей нефти и газа: дис. ... канд. тех. наук. - Пермь, 2014. - 151 с.

22. Иолчуев А.М., Савенюк О.В. Анализ метода борьбы с солеотложениями путем периодической закачки ингибитора солеотложений в призабойную зону пласта // Наука. Техника. Технологии (политехнический вестник). - 2018. - № 4. - С. 53-71.

23. Мищенко И.Т. Скважинная добыча нефти. - М.: Изд-во «Нефть и газ» РГУ нефти и газа им. И.М. Губкина, 2003. $816 \mathrm{c}$.

24. Повышение эффективности пенокислотных и большеобъемных селективных обработок на карбонатных месторождениях ПАО «Татнефть» / М.Х. Мусабиров, А.Ю. Дмитриева, Р.Ф. Хусаинов, Э.М. Абусалимов, Б.Г. Ганиев, Ф.З. Исмагилов // Нефтяное хозяйство. - 2019. - № 11. - С. 116-119.

Поступила 26.04.2021 г. 


\section{Информация об авторах}

Кривощеков С.H., кандидат технических наук, заместитель декана по научно-исследовательской работе Горно-нефтяного факультета, доцент кафедры геологии нефти и газа Пермского национального исследовательского политехнического университета.

Козырев Н.Д., аспирант кафедры нефтегазовых технологий Пермского национального исследовательского политехнического университета; инженер 1-й категории отдела гидродинамического моделирования филиала ООО «ЛУКОЙЛ-Инжиниринг» «ПермНИПИнефть» в г. Перми.

Вяткин К.A., аспирант кафедры нефтегазовых технологий Пермского национального исследовательского политехнического университета.

Равелев К.A., студент кафедры нефтегазовых технологий Пермского национального исследовательского политехнического университета. 
UDC 622.276 .63

\title{
COMPLEX APPROACH TO ESTIMATING THE EFFICIENCY OF THE DESIGNED HYDROCHLORIC ACID TREATMENT USING THE RESULTS OF LABORATORY STUDIES AND HYDRODYNAMIC SIMULATION
}

\author{
Sergey N. Krivoshchekov ${ }^{1}$, \\ Krivoshchekov@gmail.com
}

Nikita D. Kozyrev ${ }^{1,2}$,
Nikita.Kozyrev@pnn.lukoil.com

Kirill A. Vyatkin'1,

Kirill.Vyatkin@girngm.ru

Kirill A. Ravelev 1 ,

Kirill.Ravelev@pstu.ru

1 Perm National Research Polytechnic University,

29, Komsomolskiy avenue, Perm, 614990, Russia.

2 Branch of 000 «LUKOIL-Engineering» «PermNIPIneft» in Perm, 29, Sovetskoy Armii street, Perm, 614000, Russia.

The relevance of scientific work is caused by the fact that at present a high proportion of hydrochloric acid treatments do not provide the expected result in the oil fields of the Perm krai. This problem is directly related to the current approach to the design of technological operations to stimulate oil flow and insufficient knowledge of most of the factors that significantly affect the efficiency of treatment of the bottomhole zone with acid compositions. The demand for improving technologies for acid stimulation of the bottomhole zone of carbonate reservoirs and methods for predicting its effectiveness is caused by the low cost of this operation and the possibility of achieving high well productivity indicators with high-quality design and operation.

The main aim of the research is to develop an integrated approach for predicting the technological effect of the projected hydrochloric acid treatment based on a combination of laboratory research results and hydrodynamic modeling.

Objects of the research are technologies for enhancing oil recovery of reservoirs, lithologically represented by carbonate varieties. Within the main oil and gas provinces of Russia, most of the fields are at the final stage of development, as a result of which there is a low productivity of wells. Therefore, the improvement of methods for enhancing oil recovery is an important and relevant area of research in the oil and gas industry.

Methods: laboratory studies, consisting in determining the carbonate content of the studied sediments, as well as the properties of acid compositions used when carrying out hydrochloric acid treatments at the deposits of the Perm krai. These studies are necessary to establish the numerical value of the change in the skin factor on the objects under consideration. Hydrodynamic modeling taking into account this skin factor to determine the technological efficiency of the projected technology for treating the bottomhole zone of the productive formation.

Results. Based on the results of this study, there is a high correspondence between the actual changes in the skin factor at 18 objects after acid treatment, obtained from the analysis of the databases of hydrodynamic studies of wells in the Perm krai, and the predicted values according to the described method of preliminary assessment of the technological effect of hydrochloric acid treatment. The paper introduces the comparative analysis of the actual values of the target well flow rate, recorded during five months of operation, and predicted ones using hydrodynamic modeling of the fully described acid stimulation design, based on the calculated value of the skin factor change. Based on the analysis results, it was found that the proposed comprehensive approach to predicting the effectiveness of hydrochloric acid treatment has a small error within $5 \%$. Using a hydrodynamic model, the technological effect of the projected acid treatment at the target object until 2030 was calculated and a conclusion was made about the success of this geological and technical measure. With the introduction of the proposed integrated approach to oil companies, it is possible to increase the effectiveness of measures to increase oil recovery.

Key words:

Hydrochloric acid treatment, complex approach, hydrodynamic simulator, skin factor, effectiveness of measures.

The research was financially supported by the Ministry of Science and Higher Education of the Russian Federation (project no. FSNM-2020-0027).

\section{REFERENCES}

1. Khizhnyak G.P., Amirov E.A., Gladkikh E.A., Kolesov V.A., Zakharyan A.G., Kharris R. Study of the acid-generating composition effect on the cores of the productive deposits of the Kuyumbinskiy license area. Oil Industry, 2015, no. 3, pp. 31-35. In Rus.
2. Singh R., Tong S., Panthi K., Mohanty K. Stimulation of calciterich shales using nanoparticle-microencapsulated acids. SPE Journal, 2019, vol. 24, pp. 2671-2680.

3. Ridner D., Frick T., Zhu D., Hill A.D., Angeles R., Vishnumolakala N., Shuchart C.E. Influence of transport conditions on optimal injection rate for acid jetting in carbonate reservoirs. SPE Production and Operations, 2019, vol. 35, pp. 137-146. 
4. Sarmah A., Farid Ibrahim A., Nasr-El-Din H.A., Jackson J. A novel cationic polymer system that improves acid diversion in heterogeneous carbonate reservoirs. SPE Oil and Gas India Conference and Exhibition. Mumbai, 2019, pp. 2281-2295.

5. Moid F., Rodoplu R., Nutaifi A.M., Kayumov R. Acid stimulation improvement with the use of new particulate base diverter to improve zonal coverage in HPHT carbonate reservoirs. International Petroleum Technology Conference. Dhahran, 2020.

6. Aidagulov G., Gwaba D., Kayumov R., Sultan A., Aly M., Qiu X., Almajed H., Abbad M. Effects of pre-existing fractures on carbonate matrix stimulation studied by large-scale radial acidizing experiments. SPE Middle East Oil and Gas Show and Conference. Manama, 2019.

7. Hawkins M.F. A note on the skin effect. Journal of Petroleum Technology, 1956, vol. 8, pp. 65-66.

8. Ali M.T., Ezzat A.A., Nasr-El-Din H.A. A Model to simulate matrix-acid stimulation for wells in dolomite reservoirs with vugs and natural fractures. SPE Journal, 2019, vol. 25, pp. 609-631.

9. Hall-Thompson B., Ernesto A.R., Abdulrahman N., Alsuhaimi A. Acid stimulation-best practices for design, selection and testing of acid recipes in low permeability carbonate reservoirs. International Petroleum Technology Conference. Dhahran, 2020.

10. Abdrazakov D., Ziauddin M., Vernigora D., Beletskaya A., Yakimchuk I., Olennikova O., Usoltsev D., Nikolaev M., Panga M., Burlibayev A. Integration of latest laboratory, software and retarded acid technologies to increase efficiency of acid treatments in carbonates: case studies from Central Asia. International Petroleum Technology Conference. Beijing, 2019.

11. Martyushev D.A. Laboratory studies of acid compositions for treating reservoir, characterized by various carbonate content and void structure of rocks. Bulletin of the Tomsk Polytechnic University. Geo Assets Engineering, 2018, vol. 329, no. 4, pp. 6-12. In Rus.

12. Gurbatova I.P., Plotnikov V.V., Popov N.A., Sysoev I.V. Peculiarities of research of filtration characteristics of the oriented core from complex carbonate reservoirs. Bulletin of the Perm National Research Polytechnic University. Geology. Oil and Gas and Mining, 2013, no. 9, pp. 19-28. In Rus.

13. Kanevskaya R.D., Novikov A.V. Methods of wormholes simulation under hydrochloric acid impact on carbonate formations. Oilfield engineering, 2018, no. 3, pp. 19-28. In Rus.

14. Trushin Y., Aleshchenko A., Danilin K., Folomeev A., Haydar A. Gorin A., Sharifullin A. Complex approach to the design of acid treatment of carbonate reservoirs. SPE Russian Petroleum Technology Conference. Moscow, 2019.
15. Khuzin R., Shevko N., Melnikov S. Improving well stimulation technology based on acid stimulation modeling, lab and field data integration. SPE Russian Petroleum Technology Conference. Moscow, 2019.

16. Buijse M., Glasbergen G. A semi-empirical model to calculate wormhole growth in carbonate acidizing. SPE Annual Technical Conference and Exhibition. Dallas, 2005, pp. 3539-3552.

17. Daccord G., Touboul E., Lenormand R. Carbonate acidizing: toward a quantitative model of the wormholing phenomenon. SPE production engineering, 1989, vol. 4, pp. 63-68.

18. Vorobiev A.Kh. Diffuzionnye zadachi $v$ khimicheskoy kinetike [Diffusion tasks in chemical kinetics]. Moscow, Moscow University Publ., 2003. 98 p.

19. Chordia M., Trivedi J.J. Diffusion in naturally fractured reservoirs - a review. SPE Asia Pacific Oil and Gas Conference and Exhibition. Brisbane, 2010, pp. 1906-1937.

20. Ravelev K.A. Sravnitelny analiz effektivnosti primeneniya kislotnykh sostavov dlya provedeniya solyano-kislotnoy obrabotki prizaboynoy zony karbonatnogo plasta [Comparative analysis of effectiveness of acid compositions application for hydrochloric acid treatment of bottomhole zone of a carbonate reservoir]. Problemy razrabotki mestorozhdeniy uglevodorodnykh $i$ rudnykh poleznykh iskopayemykh [Problems of development of hydrocarbon and ore mineral deposits]. Perm, Perm National Research Polytechnic University Publ., 2019. pp. 194-196.

21. Potekhin D.V. Optimizatsiya tekhnologii mnogovariantnogo trekhmernogo geologicheskogo modelirovaniya zalezhey nefti $i$ gaza. Diss. Kand. nauk [Optimization of the technology of multivariate three-dimensional geological modeling of oil and gas deposits. Cand. Diss.]. Perm, 2014. 151 p.

22. Iolchuev A.M., Savenok O.V. Analysis of the method for controlling scale deposition by periodically injecting the scale inhibitor into the bottomhole formation zone. Science. Engineering. Technology (polytechnical bulletin), 2018, no. 4, pp. 53-71. In Rus.

23. Mishchenko I.T. Skvazhinnaya dobycha nefti [Borehole oil production]. Moscow, «Oil and Gas» RSU of Oil and Gas nam. I.M. Gubkina Publ., 2003. 816 p.

24. Musabirov M.Kh., Dmitrieva A.Yu., Khusainov R.F., Abusalimov E.M., Ganiev B.G., Ismagilov F.Z. Efficiency improvement of foam-acid treatments and selective large-volume acidizing at carbonate reservoirs of Tatneft PJSC. Oil Industry, 2019, no. 11, pp. 116-119. In Rus.

Received: 26 April 2021.

\section{Information about the authors}

Sergey N. Krivoshchekov, Cand. Sc., Deputy Dean for research of Mining and Petroleum Faculty, associate professor, Perm National Research Polytechnic University.

Nikita D. Kozyrev, postgraduate student, Perm National Research Polytechnic University; 1st category engineer, branch of OOO «LUKOIL-Engineering» «PermNIPIneft» in Perm.

Kirill A. Vyatkin, postgraduate student, Perm National Research Polytechnic University.

Kirill A. Ravelev, student, Perm National Research Polytechnic University. 